\title{
Adsorption and catalytic ozonation of organic pollutants from bio- treated dyeing wastewater
}

\author{
Lu Zhang ${ }^{2}$, Haitao Li ${ }^{1}$, a , Jie Liu' ${ }^{1}$, Yixin Yang ${ }^{2}$ b , Yingying $\mathrm{Li}^{2}$, Dan Song ${ }^{2}$,Wei \\ $\mathrm{Xu}^{1}$, Gengzhi $\mathrm{Yu}^{2}$, Mingsheng Luo ${ }^{2}$ \\ ${ }^{1}$ CECEP Engineering Technology Research Institute, Beijing 100082, China
}

${ }^{2}$ Beijing key laboratory of fuel clean and efficient catalytic emission reduction technology, School of Chemical Engineering, Beijing Institute Petrochemical Technology, Beijing 102617, China

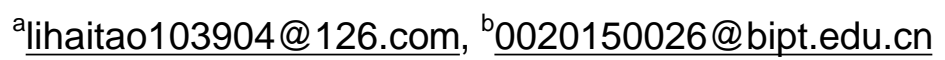

\begin{abstract}
Keywords: catalytic ozonation;dyeing wastewater;organic pollutants;GAC;adsorption
Abstract. Adsorption performance of catalysts and catalytic ozonation of organic pollutants from bio-treated dyeing wastewater were investigated. Among various catalysts, the granular activated carbon (GAC) showed the better adsorption performance for removal of COD from dyeing wastewater, and the adsorption capacity was $2.1 \mathrm{mgCOD} / \mathrm{gGAC}$. In addition, Mn@GAC composite catalyst was prepared by an impregnation method and used for catalytic ozonation.The XPS results showed that $\mathrm{Mn}$ was located onto GAC surface in the form of $\mathrm{MnO}_{2}$. The catalytic ozonation for COD removal efficiency was $41.7 \%$ with Mn@GAC. Therefore, the Mn@GAC catalytic ozonation is feasible for removal of the refractory organic pollutants from the dyeing wastewater.
\end{abstract}

\section{Introduction}

China is a huge water consumer and wastewater producer. The textile dyeing industry has always account for a large proportion about water consumption and aqueous environment pollution [1]. Effluents produced by dyeing factory are usually characterized by suspended and dissolved solids [2], smelly, strong color, chemicals, biological oxygen demand (BOD) and considerable values of chemical oxygen demand (COD), these pollutants are harmful to both the environment and human health [3]. Therefore, the discharge of printing and dyeing wastewater has faced great challenges of wastewater treatment [4].

In the textile dyeing industry, biological and physicochemical methods are commonly used to treat dyeing wastewater. The main characteristic of the bio-treated dyeing wastewater is nonbiodegradable, so biological treatments are difficult to remove the remaining organic contaminant [5]. In order to meet the discharge standards of water pollutants for dyeing and finishing of textile industry, physicochemical methods should be considered preferentially for the bio-treated dyeing wastewater treatment.

The conventional physicochemical processes for removing pollutants in dyeing wastewater contain adsorption, flocculation, membrane separation and the advanced oxidation processes (AOPs) which includes catalytic ozonation, Fenton oxidation, photocatalysis, etc [6]. Among these methods, the AOPs is mainly used for oxidizing and decomposing refractory organic pollutants by generating oxidative free radicals (hydroxyl radicals) in the reaction process [7]. The catalytic ozonation is attracting increasing attention in refractory organic wastewater treatment [8], because of its high efficiency and ambient operational conditions [9]. And various catalysts were investigated for catalytic ozonation whether play an important role in generating hydroxyl radicals and degrading pollutants, such as, active carbon (AC)[10].

This study is to investigate adsorption capacity of the catalyst and the removal efficiency of catalytic ozonation for refractory organic pollutants in bio-treated dyeing wastewater. A variety of materials were used to adsorb organic pollutant for choosing the best one as the catalyst support of catalytic ozonation. The $\mathrm{MnO}_{2}$ loaded $\mathrm{AC}$ composites was used as a catalyst in catalytic ozonation to degrade the refractory organic pollutants. The aim of this study was to evaluate the performance of catalytic ozonation on the removing of the refractory organics in bio-treated dyeing wastewater and meet the discharge standards of China. 


\section{Materials and methods}

\section{Materials and reagents}

The wastewater used for this study was obtained from a large scale dyeing wastewater plant located in southeast China. The effluent of this dyeing wastewater plant was processed by bioprocess and Flocculation precipitation technology. The main composition of dyeing wastewater is listed in Table 1.

Table 1. The main composition of dyeing wastewater

\begin{tabular}{cccccc}
\hline Parameter & $\mathrm{COD}(\mathrm{mg} / \mathrm{L})$ & $\mathrm{pH}$ & $\mathrm{TN}(\mathrm{mg} / \mathrm{L})$ & $\mathrm{TP}(\mathrm{mg} / \mathrm{L})$ & $\mathrm{EC}(\mathrm{mS} / \mathrm{cm})$ \\
\hline wastewater & $90 \sim 100$ & 8.54 & 2.58 & 1.03 & 4.61 \\
\hline
\end{tabular}

The columnar activated carbon (CAC) and GAC were obtained from Jiangsu Shengkang Technology Co., Ltd. The Y-zeolite, ZSM-5, ZSM-22, ZSM-35, SAPO-34 and mordenite were obtained from Hubei Shentan Enviromental Material Co., Ltd.

All of the chemicals used were analytical grade or above. Potassium permanganate and nitric acid were purchased from Beijing Chemical Works, China. The COD analysis of pharmaceutical products were purchased from the company of Beijing Lianhua Technology, China. All the solutions were prepared with twice distilled water.

\section{Preparation of catalysts}

The Mn@GAC composite catalyst was prepared by an impregnation method. The synthesis conditions were using precursor of potassium permanganate solution to load the surface of GAC. Briefly, as a support for the catalyst, GAC was washed with distilled water to remove the impurities and dried in a drying oven for $12 \mathrm{~h}$. Then, the GAC was submerged in potassium permanganate solution in a shaker for one day so that catalyst can be diffused into the GAC channels as much as possible. Finally, the GAC was stood for two days, washed with distilled water, dried and stored in a desiccator until used.

\section{Experimental procedure}

To compare the adsorption performance of the various catalysts for COD, the adsorption tests were performed $30 \mathrm{~min}$ by allowing $50 \mathrm{~g}$ adsorbent with $100 \mathrm{~mL}$ wastewater in $250 \mathrm{ml}$ triangle bottle on a shaker (Guohua company THZ-82A, China) operated at $25^{\circ} \mathrm{C}$ and $120 \mathrm{r} / \mathrm{min}$ [11].

To determine the adsorption capacity of the GAC for COD, the adsorption tests were performed $30 \mathrm{~min}$ by changing the dosage of GAC with $100 \mathrm{~mL}$ wastewater in a container on a shaker operated at $25^{\circ} \mathrm{C}$ and $120 \mathrm{r} / \mathrm{min}$.Langmuir were used to express the adsorption of GAC. The Langmuir model assumes the monolayer coverage of adsorbate on a homogeneous surface [12].

At the beginning of the catalytic ozonation experiment, $100 \mathrm{~mL}$ wastewater and $10 \mathrm{~g}$ catalysts were quickly added into the three-phase reactor. After that, below the gas distributor, the ozone $(1.0-1.3 \mathrm{mg} / \mathrm{min})$ from the ozonator (DHX-IX, China) was continuously fed into the reactor and the reaction was initiated. At a certain time intervals, samples were taken and filtered through a PES filter (pore size $0.45 \mu \mathrm{m}$ ) for analysis [13].

\section{Analytical and characteristic methods}

The COD of the wastewater was analyzed by COD rapid analyzer (Lianhua Technology 5B-3C (V8), China) and the theory was potassium dichromate method. The form of manganese on the catalyst was characterized by XPS (Thermo Fisher ESCALAB 250Xi, England). The content of manganese element was measured by ICP-OES (Thermo Icap 6300, America) with nitric acid pretreatment. 


\section{Results and discussion}

\section{Adsorption performance of various catalysts}

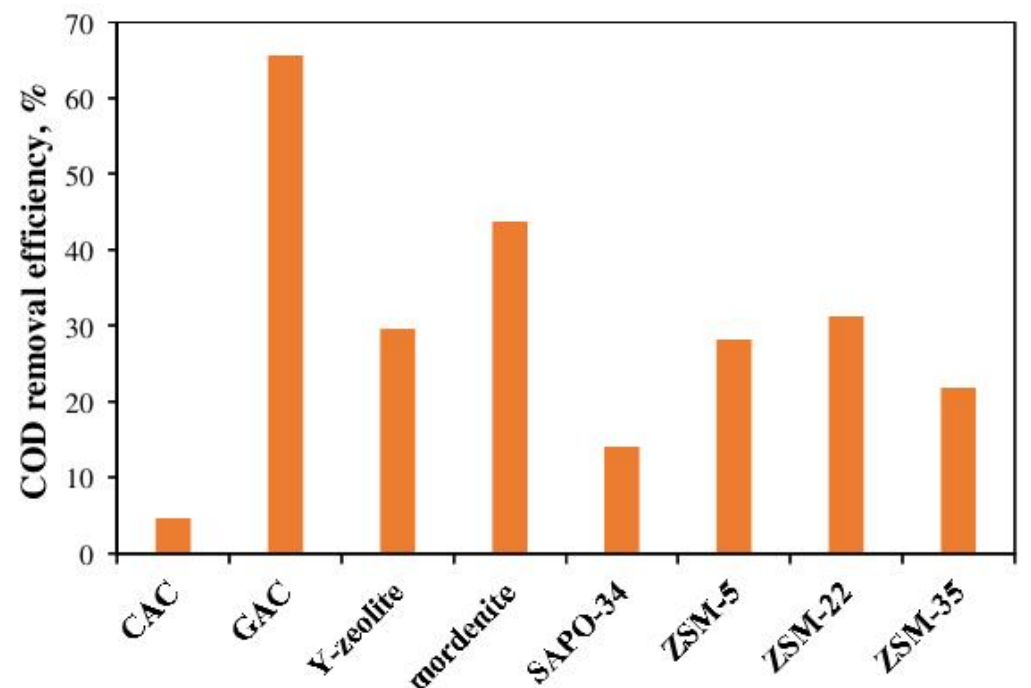

Fig. 1 The effect of various catalysts on the COD removal efficiency

Performance of various catalysts on adsorption of the dyeing wastewater was showed in Fig. 1. Compared with the other catalysts, GAC showed the best performance for the removal efficiency of COD. And the removal efficiency of COD using GAC reached $65.6 \%$ in $30 \mathrm{~min}$. Besides, The GAC is the cheapest adsorbent among them. Therefore, the adsorbent of GAC was feasible for COD adsorption in practical application [14].

\section{The characterization of the Mn@GAC catalyst}

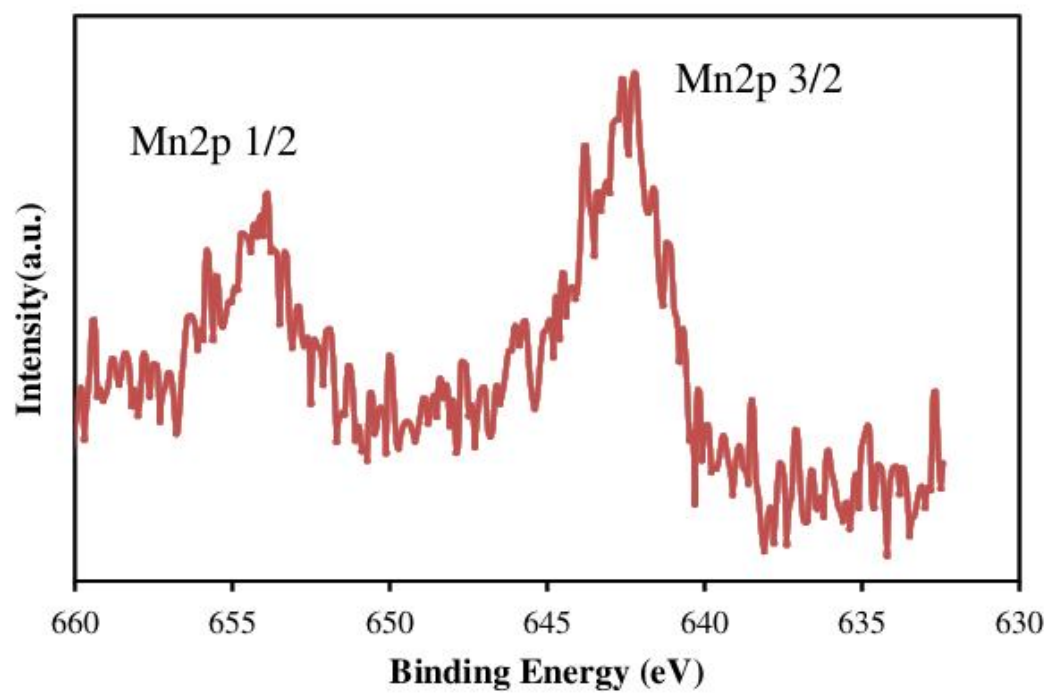

Fig. 2 XPS spectrum of Mn 2p loaded on the GAC surface

XPS spectrum was used to characterizing that Mn chemical valence on the surface of the GAC in Fig. 2. The XPS spectrum showed two peaks at 642.2 and $653.9 \mathrm{eV}$ correspond to $\mathrm{Mn} 2 \mathrm{p} 3 / 2$ and Mn 2p1/2, respectively, with a spin-energy separation of $11.7 \mathrm{eV}$, which was similar to $11.8 \mathrm{eV}$ reported by other researchers [10]. These results indicated that manganese oxide existed in the form of $\mathrm{MnO}_{2}$ on the $\mathrm{Mn} @ \mathrm{GAC}$ catalyst. Otherwise, the ICP result indicated that the weight percentage of $\mathrm{Mn}$ is $0.463 \mathrm{mg} / \mathrm{g}$ onto GAC. Otherwise, ICP result indicated that the weight percentage of Mn is $0.463 \mathrm{mg} / \mathrm{g}$ onto GAC. 


\section{Effect of GAC dosage and adsorption isotherms}
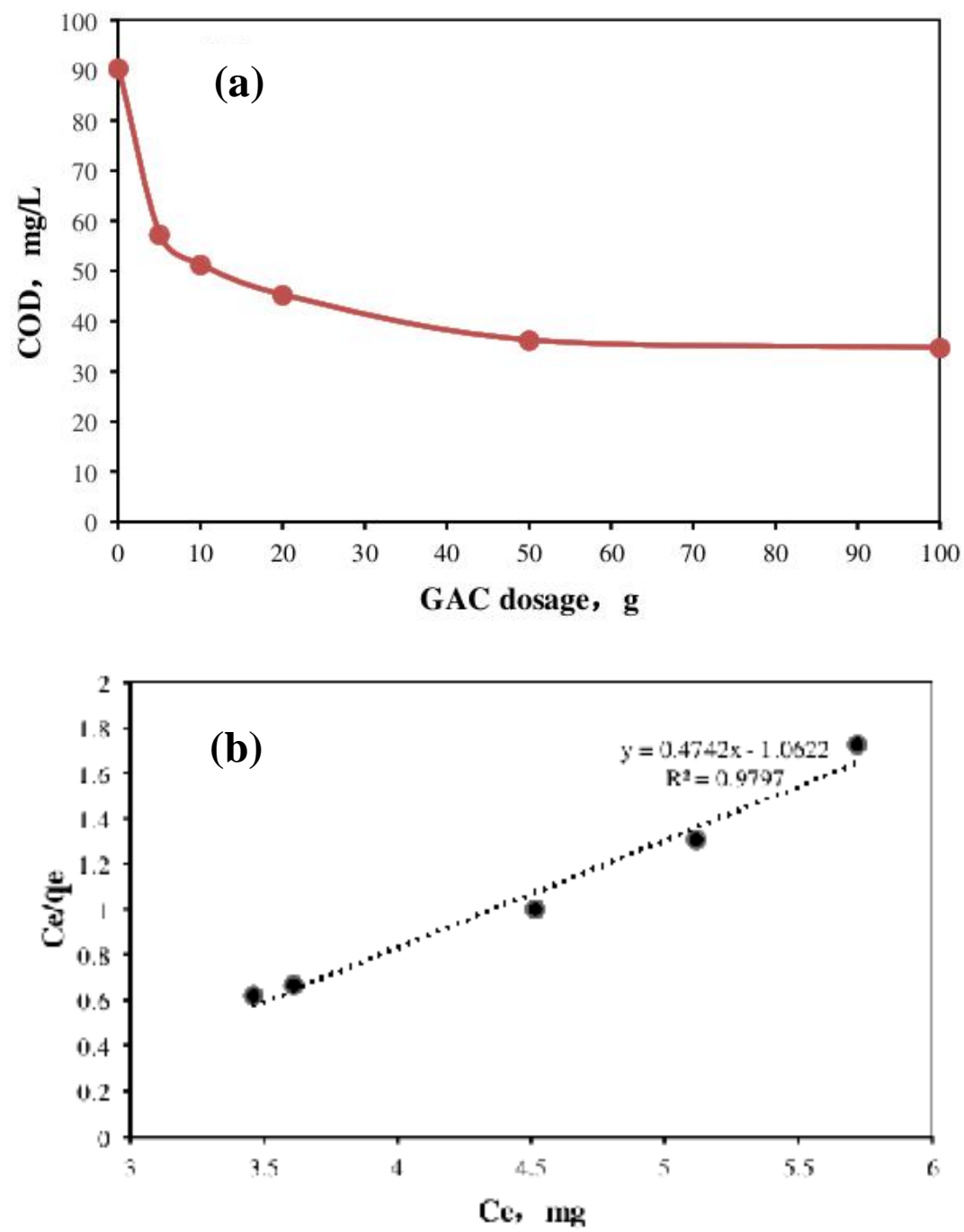

Fig. 3 The effect of GAC dosage on COD concentration in the wastewater (a) and Langmuir isotherm for COD adsorption by GAC (b)

The adsorption property of GAC for COD was investigated through an adsorptive experiment in Fig. 3(a). With the increase of GAC dosage, the COD in the wastewater decreased rapidly and then slowly. Significantly, GAC performed the excellent adsorption property of removal COD, and the COD concentration decreased from $90.3 \mathrm{mg} / \mathrm{L}$ to $34.61 \mathrm{mg} / \mathrm{L}$. The relation between Ce/qe versus Ce of the Langmuir model was linear relation with a good correlation coefficient $\left(\mathrm{R}^{2}=0.9797\right)$ for GAC (Fig. 3(b)). The maximum removal capacity (qm) of COD by GAC is $2.1 \mathrm{mg} / \mathrm{g} \mathrm{[12].}$ 


\section{Catalytic ozonation experiment}

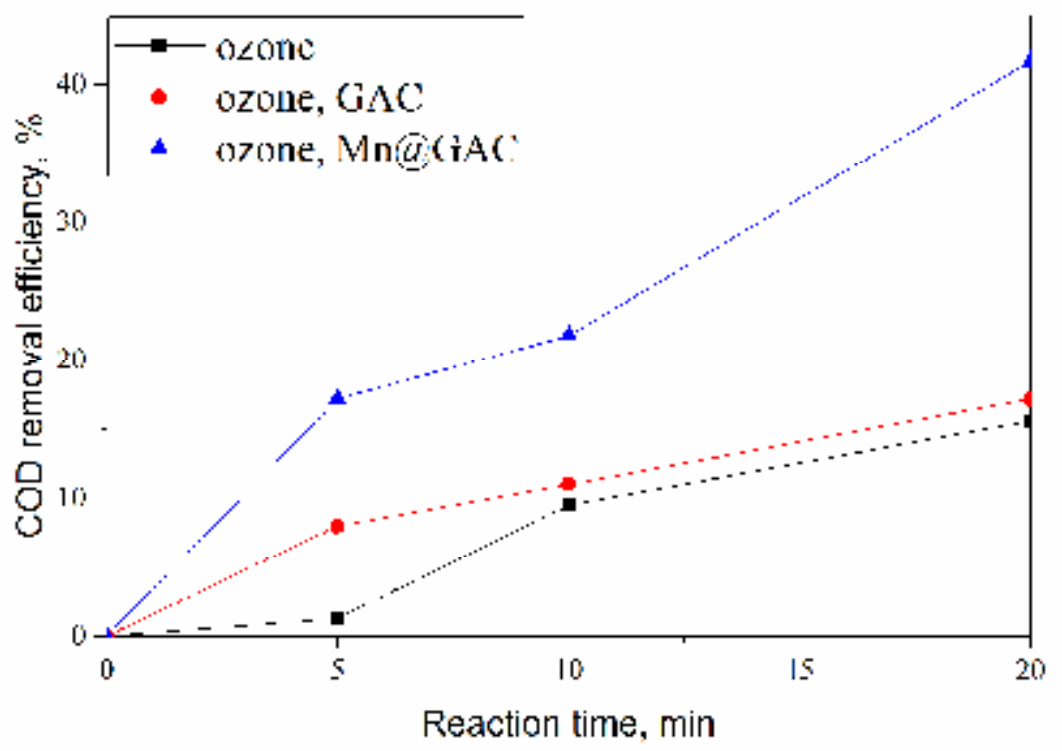

Fig. 4 Catalytic ozonation for COD removal from dyeing wastewater

As shown in Fig. 4, the COD removal efficiency increased with increasing the reaction time of catalytic ozonation. During the initial $20 \mathrm{~min}$, 41.7\% of COD was removed by the catalytic ozonation with Mn@GAC catalyst, while using GAC as a catalyst the COD removal efficicency was only $17.2 \%$ in same condition. Therefore, $\mathrm{MnO}_{2}$ on GAC as a catalyst improved the reaction rate in catalytic ozonation, resulting in a higher COD removal efficiency. When ozone was used individually, COD removal efficiency was $15.6 \%$ lower than ozone with catalysts in 20 min, so the introduction of Mn@GAC catalyst in ozonation process for dyeing wastewater can promote the ozone utilization efficiency and save the cost of wastewater treatment.

\section{Conclusions}

Among the various materials, the GAC showed the best adsorption performance for dyeing wastewater, whose maximum adsorption capacity was $2.1 \mathrm{mg} \mathrm{COD} / \mathrm{g} \mathrm{GAC}$, and the removal efficiency of COD reached $65.6 \%$ in $30 \mathrm{~min}$. The XPS spectrum displayed that the surface of Mn@GAC was existed manganese dioxide $\mathrm{MnO}_{2}$ and the result of ICP showed the weight percentage of $\mathrm{Mn}$ was $0.463 \mathrm{mg} / \mathrm{g}$. The catalytic ozonation experimental results revealed that the catalyst of Mn@GAC performed better of COD removal efficiency than ozone with GAC and ozone alone, resulting in COD removal efficiency of $41.7 \%$ in $20 \mathrm{~min}$. Therefore, the Mn@GAC catalytic ozonation is feasible for removal of the refractory organic pollutants from the dyeing wastewater.

\section{Acknowledgement}

The research was supported by the Program of Beijing Natural Science Foundation (No. 8152015) and CECEP Science and Technology Innovation Project (No. CECEP-KJ-2016-007).

\section{References}

[1] Wu, J., Ma, L., Chen, Y., Cheng, Y., Liu, Y. and Zha, X. Catalytic ozonation of organic pollutants from bio-treated dyeing and fi nishing wastewater using recycled waste iron shavings as a catalyst : Removal and pathways. Water Research, (2016), 92, 140-148.

[2] Esteves, B. M., Rodrigues, C. S. D., Boaventura, R. A. R., Madeira, L. M. and Maldonado-h, F. J. Coupling of acrylic dyeing wastewater treatment by heterogeneous Fenton oxidation in a 
continuous stirred tank reactor with biological degradation in a sequential batch reactor. Journal of Environmental Management, (2016), 166, 193-203.

[3] Rajasimman, M., Babu, S. V. and Rajamohan, N. Journal of the Taiwan Institute of Chemical Engineers Biodegradation of textile dyeing industry wastewater using modified anaerobic sequential batch reactor - Start-up , parameter optimization and performance analysis. Journal of the Taiwan Institute of Chemical Engineers, (2017), 72, 171-181.

[4] Wu, H., Liu, Z., Li, A. and Yang, H. Evaluation of starch-based fl occulants for the fl occulation of dissolved organic matter from textile dyeing secondary wastewater, Chemosphere, (2017), $174,200-207$.

[5] Tan, Y., Sun, L., Li, B., Zhao, X., Yu, T., Ikuno, N. and Hu, H. Fouling characteristics and fouling control of reverse osmosis membranes for desalination of dyeing wastewater with high chemical oxygen demand. Desalination, (2017), 419, 1-7.

[6] Zhou, T., Lu, X., Wang, J., Wong, F.-S. and Li, Y. Rapid decolourization and mineralization of simulated textile wastewater in a heterogeneous Fenton like system with/without external energy. J. Hazard. Mater. (2009), 165, 193-199.

[7] Li, Y., Li, D., Li, J., wang, J., Hussain, A., Ji, H. and Zhai, Y. Pretreatment of cyanided tailings by catalytic ozonation with $\mathrm{Mn}^{2+} / \mathrm{O}_{3}$. Journal of Environmental Sciences (China), (2015), 28, 14-21.

[8] Ghatak, H.R. Advanced oxidation processes for the treatment of bio- recalcitrant organics in wastewater. Crit. Rev. Environ. Sci. Technol. , (2014), 44 (11), 1167-1219.

[9] Hu, E., Wu, X., Shang, S., Tao, X. M., Jiang, S. X. and Gan, L. Catalytic ozonation of simulated textile dyeing wastewater using mesoporous carbon aerogel supported copper oxide catalyst. Journal of Cleaner Production, (2016), 112, 4710-4718.

[10] Li, C., Jiang, F., Sun, D. and Qiu, B. Catalytic ozonation for advanced treatment of incineration leachate using (MnO2-Co3O4 )/AC as a catalyst. Chemical Engineering Journal, (2017), 325, $624-631$.

[11] Tan, X., Wan, Y., Huang, Y., He, C., Zhang, Z., He, Z.,Hu L, Zeng J and Shu, D. Threedimensional $\mathrm{MnO} 2$ porous hollow microspheres for enhanced activity as ozonation catalysts in degradation of bisphenol A. Journal of Hazardous Materials, (2017), 321, 162-172.

[12] Ahmed M. Zayed, Ali Q. Selim, Essam A. Mohamed, Mahmoud S.M. Abdel Wahed, Moaaz K. Seliem and Mika Sillanpää. Adsorption characteristics of Na-A zeolites synthesized from Egyptian kaolinite for manganese in aqueous solutions: Response surface modeling and optimization. Applied Clay Science, (2017), 140, 17-24.

[13] Bai, Z., Yang, Q. and Wang, J. Catalytic ozonation of sulfamethazine using $\mathrm{Ce}_{0.1} \mathrm{Fe}_{0.9} \mathrm{OOH}$ as catalyst: Mineralization and catalytic mechanisms. Chemical Engineering Journal, (2016), 300, 169-176.

[14] Cai, D., Zhang, T., Zhang, F. and Luo, X. Quaternary ammonium $\beta$-cyclodextrin-conjugated magnetic nanoparticles as nano-adsorbents for the treatment of dyeing wastewater: Synthesis and adsorption studies. Biochemical Pharmacology, (2017), http://dx.doi.org/doi:10.1016/j.jece.2017.06.001 